\title{
Achieving good oral health in children: The importance of a current, relevant and unbiased evidence base in paediatric dentistry
}

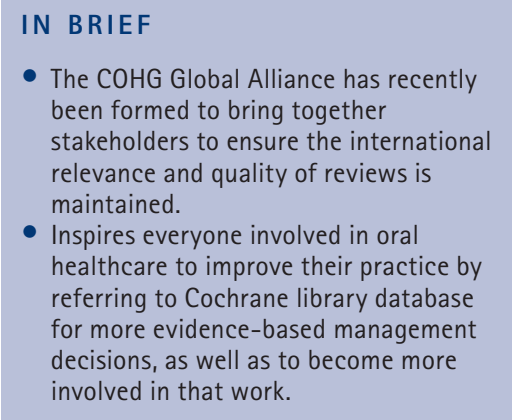

- The COHG Global Alliance has recently been formed to bring together stakeholders to ensure the international relevance and quality of reviews is maintained. (o eferring to Cochrane library database more evidence-based management nvolved in that work.

\author{
J. E. Clarkson, ${ }^{1}$ D. Bonetti ${ }^{* 2}$ and H. Worthington ${ }^{3}$
}

The Cochrane Collaboration was founded in 1993 as an international, non-profit and independent organisation dedicated to making up-to-date, accurate and reliable information about healthcare readily available. This paper discusses how the Cochrane Oral Health Group reviews have contributed to the oral health evidence base used in the development of many international and UK dental guidance documents, particularly in the field of paediatric dentistry.

\section{INTRODUCTION}

A major challenge for every dentist is determining the best evidence currently available for even the most common conditions or symptoms they treat. An inordinate amount of often contradictory research findings and 'expert' opinion is now easily available and much of this literature cannot qualify as best evidence, largely due to biased, inadequate and unsuitable methodologies. Implementing inappropriate treatment based on a poor understanding of best evidence may result in an avoidable negative impact on the oral health of dental patients.

The Cochrane Collaboration was founded in 1993 as an international, non-profit and independent organisation dedicated to making up-to-date, accurate and reliable information about healthcare readily available. It aims to help clinicians, researchers, purchasers, and patients make well-informed decisions about developing, implementing, and receiving healthcare, based on current best evidence from clinical trials and other intervention studies. ${ }^{1}$

Director, ${ }^{2}$ Senior Research Fellow, DHSRU, DDEC Frankland Building, University of Dundee, Small's Wynd, Dundee, DD1 4HN; ${ }^{3}$ Cochrane Oral Health Group School of Dentistry, The University of Manchester, Coupland 3 Building, Oxford Road, Manchester, M13 9PL

${ }^{*}$ Correspondence to: Dr Debbie Bonetti

Email: d.I.bonetti@dundee.ac.uk; Tel: 01382381702

\section{Refereed Paper}

Accepted 23 April 2013

DOI: 10.1038/sj.bdj.2013.526

${ }^{\circledR}$ British Dental Journal 2013; 214: 583-585
The Cochrane collaboration has a distinctive logo (Fig. 1) and this is explained in the following extract from the website:

'The Cochrane Collaboration logo illustrates both our organisation's global objectives and key scientific processes. The circle framed by the ' $\mathrm{C}$ ' of Cochrane and the mirror image ' $\mathrm{C}$ ' of Collaboration reflects the international collaboration that makes our work relevant globally. The inner part of the logo illustrates a systematic review of data from seven randomised controlled trials (RCTs), comparing one healthcare treatment with a placebo. Each horizontal line represents the results of one trial (the shorter the line, the more certain the result); and the diamond represents their combined results. The vertical line indicates the position around which the horizontal lines would cluster if the two treatments compared in the trials had similar effects; if a horizontal line touches the vertical line, it means that that particular trial found no clear difference between the treatments. The position of the diamond to the left of the vertical line indicates that the treatment studied is beneficial. Horizontal lines or a diamond to the right of the line would show that the treatment did more harm than good. ${ }^{1}$

Today, 20 years later, The Cochrane Collaboration's output of systematic reviews (numbering over 7,600 to date), published online in the Cochrane Database of Systematic Reviews (CDSR),

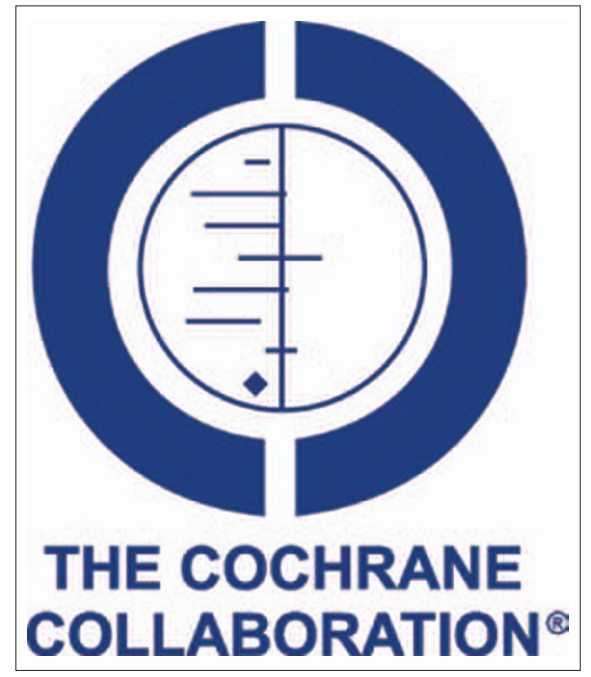

Fig. 1 The Cochrane Collaboration logo

is internationally recognised as the benchmark for high quality information about healthcare. This is due to their particularly rigorous standards in unbiased and comprehensive summarising of research evidence. The CDSR, possessing an impact factor of 5.912, is ranked in the top ten of the 155 journals worldwide listed in the 'medicine, general \&t internal' category. In 2011, the World Health Organisation (WHO) awarded the Collaboration a seat on the World Health Assembly, enabling them opportunity to inform international healthcare policy-setting.

\section{COCHRANE ORAL HEALTH GROUP}

Over 26,000 people from around the world currently contribute to The Cochrane 
Collaboration's work, carried out by 53 Cochrane Review Groups (CRGs) consisting mostly of volunteer healthcare professionals. The CRGs are supported financially by national governments, international governmental and non-governmental organisations, universities, hospitals and private donations. Commercial funding is not permitted to ensure reviews are not influenced by the interests of commercial bodies. The Cochrane Oral Health Group (COHG), along with the other UK based CRGs, receives ring-fenced funding from the National Institute for Health Research (NIHR).

The COHG, based at The University of Manchester, UK, is ranked second for its output productivity, compared with other NIHR funded CRGs. The COHG was established in 1996 in recognition of the desperate need for high quality systematic reviews of current available best evidence in dentistry. The COHG now comprises over 1,200 oral healthcare professionals, researchers and consumers from over 40 different countries around the world, publishing 132 systematic reviews, 42 review updates (which occur approximately every two years) and 66 protocols to date, with further reviews and protocols in preparation. To gauge the group's output quality, COHG's possesses an individual impact factor of 3.07 in 2011 for publication citations in its category, putting the group in the top five of dentistry journals in the world. It is not an exaggeration to say that COHG's systematic reviews comprise an oral health evidence base that is being used constructively to shape the future of dentistry, internationally as well as in the UK.

For example, COHG's reviews have contributed to the oral health evidence base used in the development of many dental guidance documents. The series of topical fluoride reviews for preventing caries in children and adolescents form the basis of the international evidence base for fluorides and are used in many guidance documents. ${ }^{2-10}$ The fissure sealant review is the core for national and international guidelines. ${ }^{11,12}$ Four reviews of oral mucositis and candidiasis have also influenced international and UK guidelines for children. ${ }^{13-16}$

The main criticism the Cochrane collaboration faces is that the reviews undertaken often raise uncertainty as to the most effective treatment/intervention etc available. The misconception is that this is due to a poor review. However, these reviews did not create inconclusive evidence base - merely reveal it. They make explicit and obvious that current treatment decisions can be based on uncertain and poor evidence. Indeed, COHG reviews revealing gaps in the oral health evidence base for routine treatments in UK dental practice has encouraged further research into fundamental aspects of oral healthcare. ${ }^{17-22}$ Currently, there are three large randomised trials in progress looking at ways of preventing or treating caries in young children, funded by the UK's National Institute of Health Research (NIHR). The FiCTION trial is examining the most clinical and cost effective approach to the management of decay in primary teeth, comparing prevention alone, conventional restorations, or sealing in decay. ${ }^{23}$ The Seal or Varnish? trial is examining whether applying fissure sealants or fluoride varnish works best, and is the most cost-effective and most acceptable from the perspective of children and their parents to prevent decay in children's teeth. ${ }^{24}$ The Northern Ireland Caries Prevention in Practice Trial (NIC-PIP) trial is looking at how effective a preventative programme (applying fluoride varnish and giving children fluoride toothpaste) is at preventing young children from developing tooth decay. ${ }^{25}$ The results of these trials should be published within the next few years and are expected to impact on policy in service and education as well as on clinician and patient decision making, not only in the UK but worldwide.

\section{GLOBAL ALLIANCE: PAEDIATRIC DENTISTRY}

Since research is always ongoing, ensuring that reviews are conducted and updated in a timely manner is a major issue for the CRGs. To that end, the COHG Global Alliance (GA) has recently been formed, comprising of experts from across the spectrum of international organisations and specialist groups in all areas of oral healthcare. The purpose of GA is to bring together stakeholders (including the British Society of Paediatric Dentistry) to increase methodological capacity at the editorial base, ensuring the international relevance as well as the quality of reviews is maintained, and to prioritise reviews and their updates.
For example, the GA is currently focusing on paediatric dentistry. A survey of all authors of Cochrane's 33 paediatric dentistry reviews (including protocols and titles) was undertaken to inform the prioritisation of review updates. These authors identified the most important top ten reviews from the full COHG list. The majority of assessors ranked the topical fluoride and sealant reviews as most important..$^{2-6,12}$ In addition to the author survey, a group of international paediatric dental experts were also asked to consider the COHG list. They also agreed that the topical fluoride and sealant reviews should be prioritised for updating. Additionally, they suggested that prioritised reviews should include community and population-based complex interventions and behavioural management strategies. This was to support their consensus that the way forward was to bring together regional variances in recommendations on the prevention of caries and develop internationally relevant guidance.

The suggestion to include reviews about complex interventions reflects an increasing awareness the importance of understanding issues concerning the implementation of research evidence. Although there is an increasing volume of literature in the area of guidance implementation and research/knowledge translation, it is universally recognised that this is a relatively neglected area. TRiaDS (Translation Research in a Dental Setting) is a programme where international researchers are now collaborating to further an understanding of knowledge translation (evidence to practice) issues, whether these concern the initial development and presentation of the evidence, guideline design, at the level of the organisation, or at the level of the individual clinician or patient. TRiaDS includes international and UK academics, doctors and dentists from primary and secondary care, psychologists, economists, statisticians, triallists and policy makers. Further information on TRiaDS can be accessed on the TRiaDS, Scottish Dental Clinical Effectiveness Programme (SDCEP), and the Scottish Dental Practice Based Research Network (SDPBRN) websites, or in the published protocol. ${ }^{26-28}$ The overall aim of this programme is to develop a generalisable framework that is readily transferable across national and international jurisdictions, and professional disciplines. 
The TRiaDS framework is being informed and informing implementation research that now occurs concurrently with the production of all clinical guidance in Scotland produced by SDCEP. For example, part of the TRiaDS protocol involves performing mixed methods diagnostic research investigating the impact of guidance publications. This protocol was followed for the SDCEP guidance on The Prevention and Management of Dental Caries in Children. ${ }^{9}$ The results of this survey ( $\mathrm{N}=131 \mathrm{GDPs}$ ) are reported on the SDPBRN website. ${ }^{28}$ In summary, the results of the survey suggest that the guidance has had little impact on the number of topical fluorides and PFSs being applied in Scotland. Participating GDPs did not intend to change their behaviour, even when they acknowledged that they were not implementing recommended practice for these treatments. The survey also revealed that participating dentists considered fluoride varnish to be a simple but relatively ineffective treatment, and that PFSs were considered to be slightly more effective than FV, but significantly more difficult to do. The results of this survey can inform future efforts to encourage the application of preventive strategies, by exploring these beliefs in more detail.

\section{CONCLUSION}

Developing an internationally relevant oral healthcare evidence base consisting of up-to-date, unbiased, reviews of the effectiveness of interventions, as produced by $\mathrm{COHG}$, is vital to all those involved in making decisions about healthcare. This includes individual dentists trying their best to ensure the quality of the care they deliver to their patients, as well as policy makers and research funders, who can use evidence from research to direct or deny investment in healthcare. It is simply not possible to achieve and further the best oral healthcare for children without such an evidence base in paediatric dentistry and a better understanding of how to translate that evidence base into practice.

If you would like to be involved in the work that COHG undertakes, please contact the editorial base at cohg@manchester. ac.uk or visit www.ohg.cochrane.org for further information.

1. The Cochrane Library [database on disk and CD ROM]. Cochrane Collaboration. Oxford: Update Software, 1996. Updated quarterly. Information available online at www.thecochranelibrary.com (accessed May 2013).

2. Marinho V C, Higgins J P, Logan S, Sheiham A. Fluoride gels for preventing dental caries in children and adolescents. Cochrane Database Syst Rev 2002; (2): CD002280.

3. Marinho V C, Higgins J P, Logan S, Sheiham A. Fluoride varnishes for preventing dental caries in children and adolescents. Cochrane Database Syst Rev 2002; (3): CD002279.

4. Marinho V C, Higgins J P, Logan S, Sheiham A. Fluoride mouthrinses for preventing dental caries in children and adolescents. Cochrane Database Syst Rev 2002; (3): CD002284.

5. Marinho V C, Higgins J P, Logan S, Sheiham A. Fluoride toothpastes for preventing dental caries in children and adolescents. Cochrane Database Syst Rev 2003; (1): CD002278.

6. Marinho V C, Higgins J P, Sheiham A, Logan S. One topical fluoride (toothpastes, or mouthrinses, or gels, or varnishes) versus another for preventing dental caries in children and adolescents. Cochrane Database Syst Rev 2004; (1): CD002780.

7. Scottish Intercollegiate Guidelines Network. SIGN 83: Prevention and management of dental decay in the pre-school child. Edinburgh: Scottish Intercollegiate Guidelines Network, 2005. Information available online at www.sign.ac.uk/ guidelines (accessed May 2013).

8. Welbury R, Raadal M, Lygidakis N A. European Academy of Paediatric Dentistry. EAPD guidelines for the use of pit and fissure sealants. Eur J Paediatr Dent 2004; 5: 179-184 (www.eapd.eu).

9. Scottish Dental Clinical Effectiveness Programme. Guidance on the Prevention and Management of Dental Caries in Children. Dundee: SDCEP, 2010. Information available online at www.SDCEP.org.uk (accessed May 2013).

10. British Association for the Study of Community Dentistry. Delivering Better Oral Health: An evidencebased toolkit for prevention. Department of Health, 2000. Report available online at www.avon.nhs.uk/ dental/publications (accessed May 2013).

11. Scottish Intercollegiate Guidelines Network. SIGN 47: Preventing dental caries in children at high caries risk: Targeted prevention of dental caries in the permanent teeth of 6-16 year olds presenting for dental care. Edinburgh: Scottish Intercollegiate Guidelines Network, 2000. Information available online at www.sign.ac.uk/guidelines (accessed May 2013).

12. Ahovuo-Saloranta $A$, Hiiri $A$, Nordblad $A$, Worthington $\mathrm{H}$, Mäkelä M. Pit and fissure sealants for preventing dental decay in the permanent teeth of children and adolescents. Cochrane Database Syst Rev 2004; (3): CD001830.

13. Worthington HV, Clarkson JE, Bryan G, Furness $S$, Glenny A-M, Littlewood A, McCabe MG, Meyer S, Khalid T. Interventions for preventing oral mucositis for patients with cancer receiving treatment. Cochrane Database of Systematic Reviews 2011; (4): CD000978.

14. Worthington HV, Clarkson JE, Khalid T, Meyer S, McCabe M. Interventions for treating oral candidiasis for patients with cancer receiving treatment. Cochrane Database of Systematic Reviews 2010; (7): CD001972.

15. Clarkson JE, Worthington HV, Furness S, McCabe $\mathrm{M}$, Khalid T, Meyer S. Interventions for treating oral mucositis for patients with cancer receiving treatment. Cochrane Database of Systematic Reviews 2010; (8): CD001973

16. Clarkson JE, Worthington HV, Eden TOB. Interventions for preventing oral candidiasis for patients with cancer receiving treatment. Cochrane Database of Systematic Reviews 2007 . (1): CD003807.

17. Ricketts D, Kidd E, Innes N P T, Clarkson J E. Complete or ultraconservative removal of decayed tissue in unfilled teeth. Cochrane Database Syst Rev 2006; (3): CD003808.

18. Innes N P T, Ricketts D, Evans D J P. Preformed metal crowns for decayed primary molar teeth Cochrane Database Syst Rev 2007; (1): CD005512.

19. Yengopal V, Mickenautsch S, Bezerra A C, Leal S C. Caries-preventive effect of glass ionomer and resinbased fissure sealants on permanent teetha meta analysis. J Oral Sci 2009; 51: 373-382.

20. Mickenautsch S, Yengopal V. Caries-preventive effect of glass ionomer and resin-based fissure sealants on permanent teeth: An update of systematic review evidence. BMC Res Notes 2011; 4: 22.

21. Beirne $P$, Worthington H V, Clarkson J E. Routine scale and polish for periodontal health in adults. Cochrane Database Syst Rev 2005; (1): CD004625.

22. Beirne P, Clarkson J E, Worthington H V. Recall intervals for oral health in primary care patients. Cochrane Database Syst Rev 2005, (2): CD004346.

23. FiCTION Filling children's teeth: indicated or not? Project information available online at www.hta. ac.uk/1783 (accessed May 2013).

24. Seal or varnish? A randomised trial to determine the relative cost and effectiveness of pit and fissure sealants and fluoride varnish in preventing dental decay. Project information available online at www. hta.ac.uk/2202 (accessed May 2013).

25. Northern Ireland Caries Prevention In Practice Trial (NIC-PIP). A randomised control trial to measure the effects and costs of a dental caries prevention regime for young children attending primary care dental services. Project information available online at www.hta.ac.uk/1970 (accessed May 2013).

26. Clarkson J E, Ramsay C R, Eccles M P et al. The translation research in a dental setting (TRiaDS) programme protocol. Implement Sci 2010; 5: 57.

27. Scottish Dental Clinical Effectiveness Programme. Information available online at www.sdcep.org.uk (accessed May 2013).

28. Scottish Dental Practice Based Research Network. Information available online at www.sdpbrn.org.uk (accessed May 2013). 